\title{
Blood-Based Circulating MicroRNAs are Potential Diagnostic Biomarkers for Leukemia: Result from a Meta-Analysis
}

\author{
Li-hua Xu ${ }^{a, b}$ Yang Guo ${ }^{b} \quad$ Xue-Li Zhang c,d Jia-jia Chen ${ }^{\mathrm{c}}$ Shao-yan Hu \\ aDepartment of Hematology and Oncology, Children's Hospital of Soochow University, Suzhou, \\ 'The First People's Hospital of Lianyungang, Lianyungang, 'Center for Systems Biology, Soochow \\ University, Suzhou, ${ }^{d}$ Department of Biochemistry and Molecular Biology, Soochow University, Suzhou, \\ eSchool of Chemistry and Biological Engineering, Suzhou University of Science and Technology, \\ Suzhou, China
}

\section{Key Words}

Circulating miRNAs $\bullet$ Leukemia $・$ Meta-analysis $・$ Diagnosis $・$ Biomarker

\begin{abstract}
Aims: Circulating microRNAs (miRNAs) as biomarkers for leukemia have been validated by emerging studies. This meta-analysis aims to estimate the overall diagnostic accuracy of bloodbased circulating miRNAs for leukemia. Methods: We searched multiple databases (PubMed, EMBASE, Cochrane Library, CNKI, Wan Fang Data and CQVIP) up to June 18, 2015. Results: 32 studies from 10 publications were included in this meta-analysis. Diagnostic capacity was evaluated by pooled sensitivity, specificity, positive likelihood ratio (PLR), negative likelihood ratio (NLR), diagnostic odds ratio (DOR), and area under the curve (AUC) through randomeffects model. Sensitivity analyses were sequentially performed to find potential sources of heterogeneity. The quality of included studies was assessed by QUADAS (quality assessment for studies of diagnostic accuracy). Meta-Disc 1.4 and Stata 12.0 software were used to perform the meta-analysis. A high diagnostic accuracy was displayed, with a sensitivity of 0.84 , a specificity of 0.88 , a PLR of 7.20 , a NLR of 0.18 , a DOR of 52 , and an AUC of 0.94 . Subgroup analyses revealed better performance for combined miRNAs, acute myeloid leukemia patients and Asian population than other subgroups. Conclusion: Our analyses suggested that bloodbased circulating miRNAs are promising diagnostic biomarkers for leukemia, especially combined miRNAs. Its clinical application awaits further study.
\end{abstract}

\section{Introduction}

Leukemia is a highly heterogeneous hematopoietic cancer, characterized by the accumulation of immature cells and disturbed hematopoiesis in bone marrow. Every year,

L.-h. Xu, Y. Guo and X.-L. Zhang contributed equally to this work.

Dr. Shao-yan $\mathrm{Hu}$

KARGER
Department of Hematology and Oncology, Children's Hospital of Soochow University, 303 Jingde Road, Suzhou, Jiangsu, (P.R. China)

E-Mail hsy139@126.com 


\section{Cellular Physiology Cell Physiol Biochem 2016;38:939-949 and Biochemistry Published online: March 04, $2016 \quad \begin{aligned} & \text { DOI: 2016 The Author(s). Published by S. Karger AG, Basel } \\ & \text { www.karger.com/cpb }\end{aligned}$ \\ Xu et al.: Circulating MiRNAs in Leukemia Diagnosis}

over 250,000 individuals worldwide are diagnosed with leukemia, which includes four major categories: acute myeloid leukemia, acute lymphocytic leukemia, chronic myeloid leukemia, and chronic lymphocytic leukemia [1]. Significant improvements in diagnosis and treatment of leukemia have been presented in the last decades, thanks to MICM (morphology, immunology, cytogenetic, molecular) diagnostic classification for leukemia since $2001[2,3]$. At the same time, the overall incidence rate of leukemia is still on the rise (by $0.5 \%$ per year) [4]. The current MICM has contributed to personalized treatment and prognostic evaluation of leukemia, but it is hyposensitive, costly, and invasive, with a complex process of bone marrow extraction. Thus, novel noninvasive biomarkers for diagnosis and classification of leukemia are desperately needed.

MicroRNAs (miRNAs) are a family of short, single strand and non-coding RNAs ( $\sim 22 \mathrm{nt})$, which target over 30\% of human genes [5]. Disturbance of miRNA expression can contribute to the multistep processes of carcinogenesis [6, 7]. Circulating miRNAs are a class of miRNAs originating from tissues and appearing in the cell-free body fluids (e.g. plasma, serum, urine, saliva, etc.). Their notable characteristics such as ease of access, high stability increase their potential as diagnostic, prognostic, and predictive biomarkers of the disease [8-10]. A recent research has indicated that disturbing expression of circulating miR-200c was significantly associated with the outcome in cancer patients [11]. In 2008, three studies covered the field on circulating miRNAs at the same time $[8,12,13]$, which prompted the investigation on circulating miRNAs as novel and minimal invasive biomarkers of cancers. The first reported blood-based circulating miRNA was miR-21, which aberrantly expressed in serum of patients with diffuse large B-cell lymphoma [14]. Since then, increasing studies have identified growing number of circulating miRNAs as potential diagnostic biomarkers for leukemia [15-18].

However, many discrepancies are presented among these findings. Xie et al. first reported the up-regulation of serum miR-155 in patients with AML, with a diagnostic accuracy of sensitivity $69 \%$ and specificity $88 \%$ [19]. Subsequently, Zhi et al. also examined circulating miR-155 in AML patients but reached at relatively higher sensitivity and specificity of $90 \%$ and 96\%, respectively [18]. This inconsistency awaits more interpretation. Furthermore, another study examined the performance of two miRNAs (miR-150 and miR-155) in distinguishing acute leukemia (AL) from healthy controls, and found that their combination had a higher diagnosis accuracy than single ones [20]. In addition, Ohyashiki et al. found that the diagnostic accuracy of miR-92a was significantly elevated using cell to plasma ratio of miR-92a in patients with AL [21]. These findings suggest that miRNAs in peripheral blood as diagnostic biomarkers for leukemia is desirable under certain improvements. Differences among studies need further systematic evaluation. Hence, we performed the present metaanalysis, to provide an up-to-date and comprehensive assessment on the overall diagnostic accuracy of blood-based circulating miRNAs for leukemia.

\section{Material and Methods}

Search strategy and study selection

We performed comprehensive search in diverse databases, including the PubMed, Cochrane library, EMBASE, Chinese National Knowledge Infrastructure (CNKI), Wan Fang Data and Technology of Chongqing databases (CQVIP) until June18, 2015, but without a lower date and language limit. The search terms were "microRNA or miRNA or has-miR", "circulating or serum or sera or plasma or peripheral blood", "leukemia or hematological malignancy/cancer/ tumor/carcinoma/neoplasms", and "diagnosis or sensitivity or specificity or ROC curve". In addition, to identify additional information, we also reviewed the references list in retrieved studies, and even contacted authors if necessary.

Further inclusion criteria for the meta-analysis were as follows: (1) clinical studies on evaluation of blood-based circulating miRNAs in diagnosis of leukemia; (2) studies presenting complete data to allow construction of fourfold table; (3) studies using healthy individuals as controls; (4) original research papers. The exclusion criteria included: (1) studies without sufficient data; (2) unqualified data; (3) duplicate 


\section{Cellular Physiology Cell Physiol Biochem 2016;38:939-949 \\ \begin{tabular}{l|l} 
and Biochemistry Published online: March 04, 2016 & $\begin{array}{l}\text { Do } 2016 \text { The Author(s). Published by S. Karger AG, Basel } \\
\text { www.karger.com/cpb }\end{array}$
\end{tabular} \\ Xu et al.: Circulating MiRNAs in Leukemia Diagnosis}

publication; (4) studies with patients less than 20. Study eligibility was assessed independently by two reviewers (Lihua Xu and Yang Guo). Another reviewer (Xueli Zhang) also joined in data extraction. Any disagreements were resolved under full discussion with the third reviewer.

\section{Data extraction and quality assessment}

Two independent reviewers screened literatures for the following data: first author, year of publication, ethnicity, disease type, sample size, specimen source, miRNAs profile, test method, sensitivity and specificity. Quality Assessment of Diagnostic Accuracy Studies (QUADAS) checklist was employed to evaluate the quality of included studies [22].

\section{Statistical analysis}

According to the guidelines for diagnostic accuracy meta-analysis [23], we extracted accuracy data from each study, including true positives (TP), false positives (FP), true negatives (TN), and false negatives (FN). Sensitivity, specificity, positive likelihood ratio (PLR), and negative likelihood ratio (NLR) were calculated to evaluate the ability to distinguish leukemia patients from the healthy controls. Diagnostic odds ratio (DOR) and area under the curve (AUC) of the summary receiver operator characteristic (SROC) curve were used to assess overall performance of each diagnostic test. The heterogeneity among studies was assessed by index $\left(\mathrm{I}^{2}\right)$ of pooled DOR. $\mathrm{I}^{2}$ value $>50 \%$ indicated the existence of heterogeneity. Statistical analyses were performed by Meta-Disc version 1.4 software [24] and Stata version 12.0 (Stata Corporation, College Station, TX, USA). All statistical tests were two sided, with significance set at $p<0.05$. Deeks' funnel plot was used to identify potential publication bias.

\section{Results}

\section{Quality and characteristics of included studies}

The study selection flowchart is showed as Fig. 1. A total of 384 articles were retrieved through the above database $(n=378)$ and other sources $(n=6) .320$ articles were excluded on the basis of title and abstract. The full texts of the remaining 64 articles were carefully screened and 54 articles failing to meet the inclusion criteria were excluded. Finally, ten highquality articles were used in this meta-analysis, of which, three were on AL (not separated clearly for ALL or AML), five were on AML, two were on CLL. Ten articles contained 32 studies which were all brought into our analyses [15-21, 25-27], including 554 patients with leukemia and 409 healthy controls. The main information of included studies was listed in Table 1. The QUADAS scores ranged from 10 to 13 , suggesting high quality of the included studies.

\section{Diagnostic accuracy analysis}

Forest plot of sensitivity and specificity for blood-based circulating miRNA in the diagnosis of leukemia were showed in Fig. 2. The summary estimates for diagnostic accuracy were listed in Table 2 . Pooled sensitivity, specificity, PLR, and NLR were $0.84,0.88,7.20,0.18$ (Fig. 2a-d), respectively, which revealed that circulating miRNAs were great biomarkers for diagnosis of leukemia. Overall diagnostic accuracy was assessed by the pooled DOR (Fig. 2e) and AUC (Fig. 2f). Their values were 52 and 0.94 ,

Fig. 1. Flowchart of suitable studies selection process.

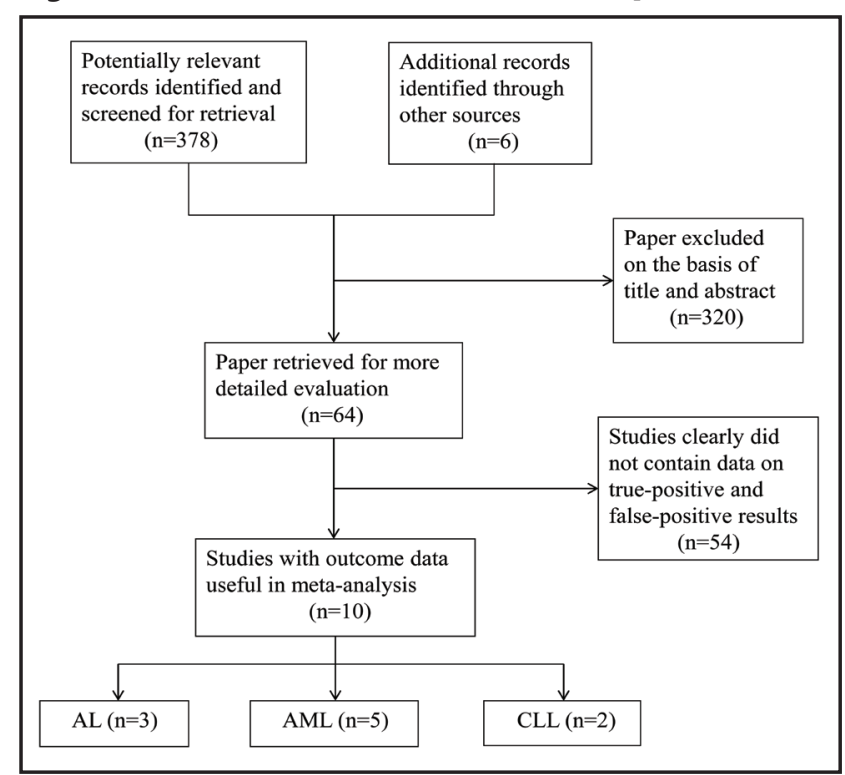




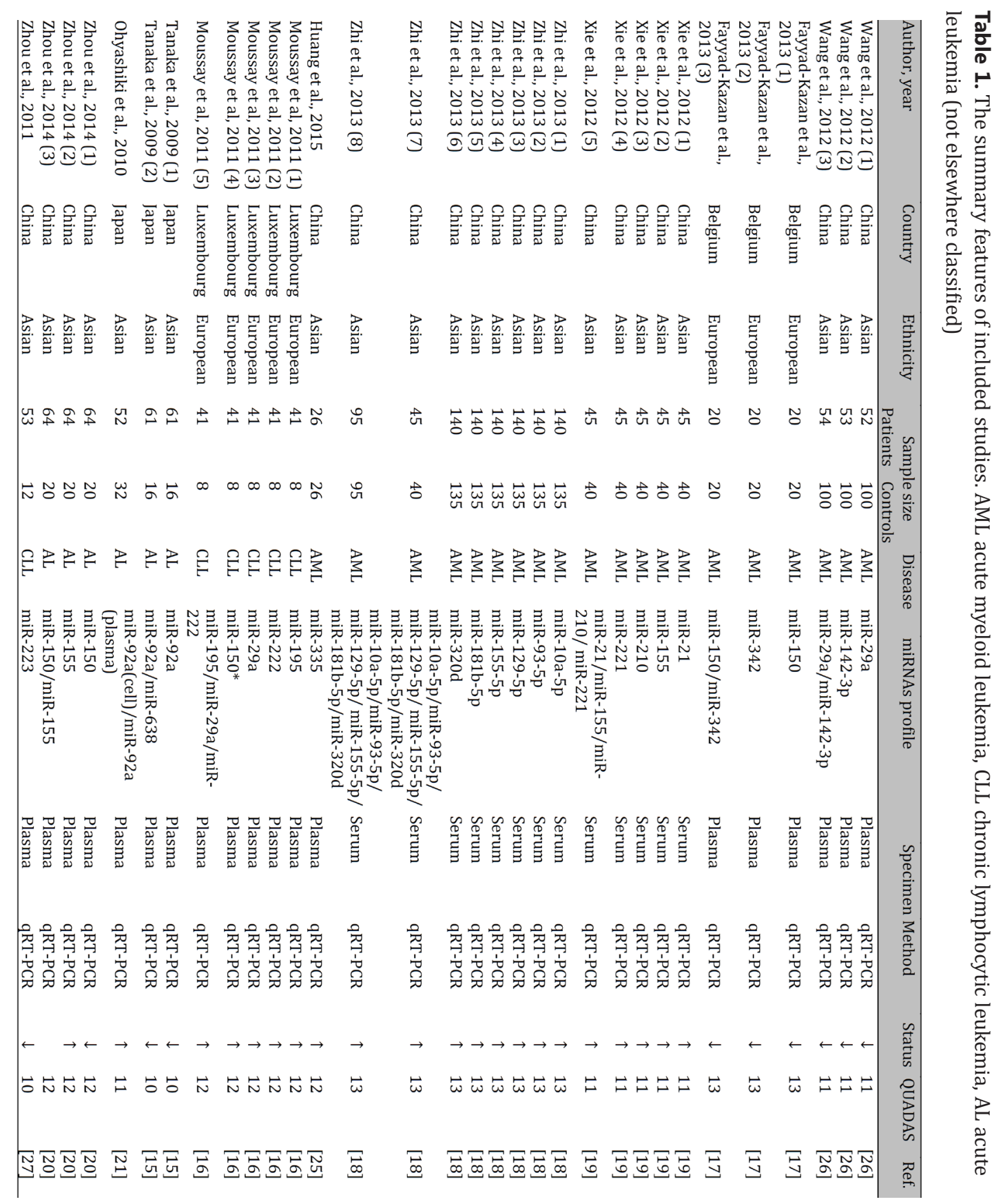

respectively, suggesting a high diagnostic accuracy. Nevertheless, the $\mathrm{I}^{2}$ value of pooled DOR was $75.7 \%$, which indicated a high heterogeneity among 32 studies. Therefore, we selected a Random-effects Model throughout the meta-analysis, and performed a series of sensitivity analysis (threshold effect, meta-regression and subgroup analyses) to seek the sources of heterogeneity.

\section{Analysis diagnostic threshold effect}

The threshold effect is a pivotal source of heterogeneity in diagnostic tests. It is caused by differences in the sensitivity and specificity. One good way to assess the threshold effect is Spearman correlation coefficient of sensitivity and specificity [24]. Our analyses showed that Spearman correlation coefficient in total 32 studies was- $0.04(\mathrm{P}=0.83)$, indicating that the heterogeneity was not caused by threshold effect. 


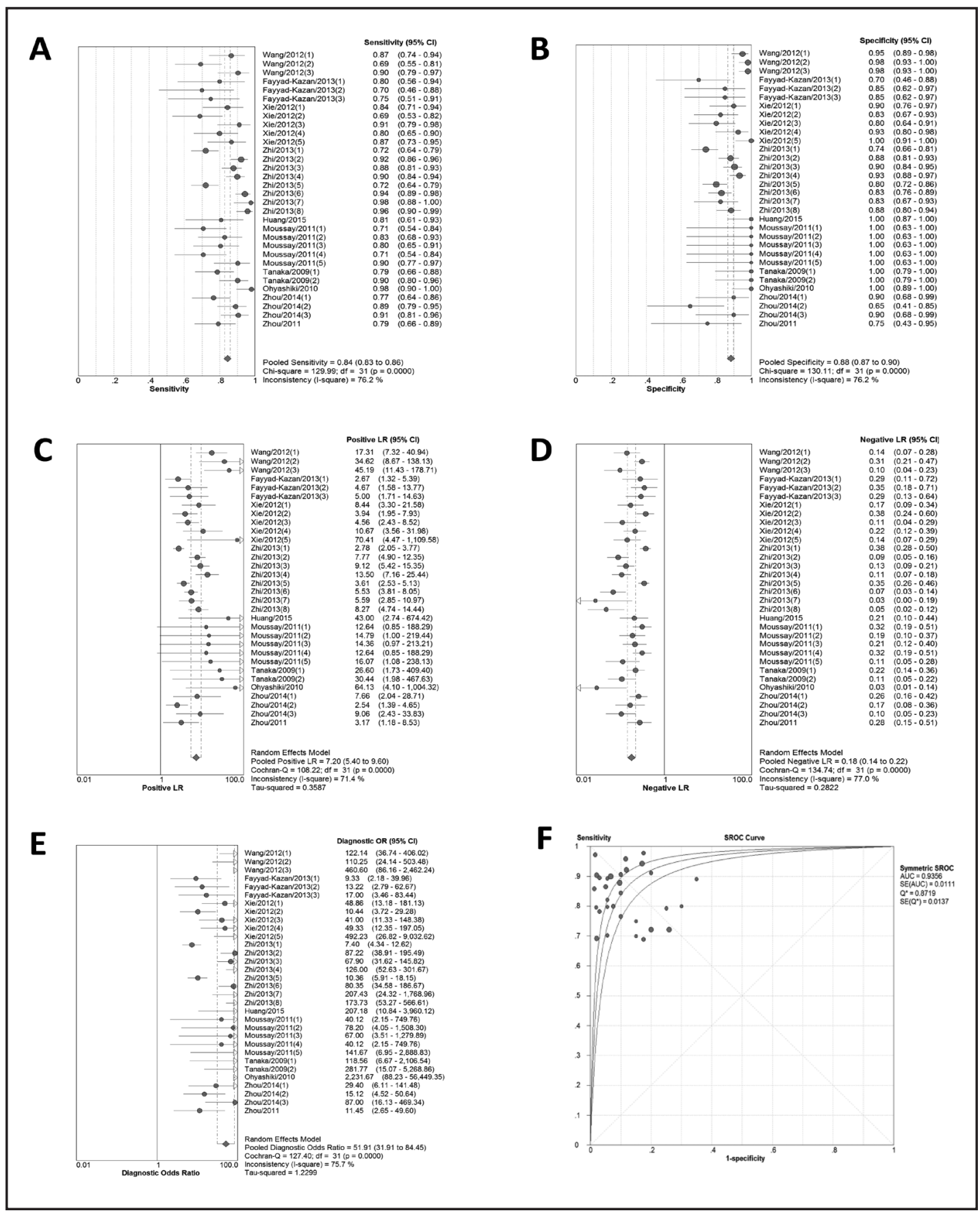

Fig. 2. Forest plot of diagnostic accuracy index of altered circulating miRNAs expression in leukemias. (A) Sensitivity of altered circulating miRNAs expression in diagnosis of leukemia in all studies. (B) Specificity of altered circulating miRNAs expression in diagnosis of leukemia in all studies. (C) Positive likelihood ratio of altered circulating miRNAs expression in diagnosis of leukemia in all studies. (D) Negative likelihood ratio of altered circulating miRNAs expression in diagnosis of leukemia in all studies. (E) Diagnostic odds ratio of altered circulating miRNAs expression in diagnosis of leukemia in all studies. (F) SROC of altered circulating miRNAs expression in diagnosis of leukemia in all studies.

Meta-regression analysis

Subsequently, meta-regression analysis was performed. We found that miRNAs profile was the main source of heterogeneity [RDOR $=4.94$ (95\%CI: 1.71-14.24), $p=0.004]$. It 


\begin{tabular}{|c|c|c|}
\hline Cellular Physiology & Cell Physiol Biochem 2016;3 & 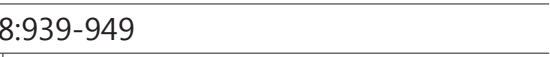 \\
\hline and Biochemistry & $\begin{array}{l}\text { DOI: 10.1159/000443046 } \\
\text { Published online: March 04, } 2016\end{array}$ & $\begin{array}{l}\text { O } 2016 \text { The Author(s). Published by S. Karger AG, Basel } \\
\text { wwu.karger.com/cpb }\end{array}$ \\
\hline
\end{tabular}

Table 2. Summary diagnostic accuracy of blood-based circulating miRNA assays for leukemia. CI confidence interval, LR likelihood ratio, DOR diagnostic odds ratio, AUC area under the curve, AL acute leukemia (not elsewhere classified), AML acute myeloid leukemia, CLL chronic lymphocytic leukemia, AL* union of AL and AML

\begin{tabular}{|c|c|c|c|c|c|c|}
\hline Analysis & $\begin{array}{l}\text { Sensitivity } \\
(95 \% \mathrm{CI})\end{array}$ & $\begin{array}{c}\text { Specificity } \\
(95 \% \mathrm{CI})\end{array}$ & $\begin{array}{c}\text { Positive LR } \\
\text { (95\%CI) }\end{array}$ & $\begin{array}{l}\text { Negative LR } \\
(95 \% \mathrm{CI})\end{array}$ & $\begin{array}{c}\text { DOR } \\
(95 \% \mathrm{CI})\end{array}$ & $\begin{array}{r}\text { AUC } \\
\text { (SEM) }\end{array}$ \\
\hline \multicolumn{7}{|l|}{ Type of patients } \\
\hline $\mathrm{AL}^{*}(\mathrm{AL}+\mathrm{AML})$ & $0.85(0.83-0.87)$ & $0.88(0.87-0.90)$ & $7.22(5.31-9.80)$ & $0.17(0.13-0.22)$ & $54(32-93)$ & $0.94(0.01)$ \\
\hline $\mathrm{AL}$ & $0.87(0.83-0.90)$ & $0.91(0.85-0.96)$ & $10.47(3.00-36.49)$ & $0.15(0.09-0.25)$ & $76(21-273)$ & $0.94(0.02)$ \\
\hline AML & $0.85(0.83-0.87)$ & $0.88(0.86-0.90)$ & $7.12(5.16-9.82)$ & $0.17(0.13-0.24)$ & $51(28-92)$ & $0.94(0.01)$ \\
\hline CLL & $0.79(0.74-0.84)$ & $0.94(0.84-0.99)$ & $5.77(2.68-12.40)$ & $0.25(0.19-0.33)$ & $30(11-79)$ & $0.88(0.05)$ \\
\hline \multicolumn{7}{|l|}{ MiRNAs profile } \\
\hline Single miRNA & $0.82(0.80-0.84)$ & $0.87(0.85-0.89)$ & $6.27(4.60-8.55)$ & $0.21(0.17-0.27)$ & $38(22-60)$ & $0.92(0.01)$ \\
\hline MiRNA panels & $0.92(0.89-0.94)$ & $0.93(0.90-0.96)$ & $11.53(6.10-21.79)$ & $0.10(0.07-0.16)$ & $165(69-392)$ & $0.97(0.01)$ \\
\hline \multicolumn{7}{|l|}{ Sample types } \\
\hline Plasma-based & $0.83(0.80-0.85)$ & $0.94(0.91-0.96)$ & $9.88(5.53-17.66)$ & $0.21(0.17-0.26)$ & $54(28-102)$ & $0.91(0.02)$ \\
\hline Serum-based & $0.86(0.84-0.88)$ & $0.86(0.84-0.88)$ & $6.20(4.49-8.57)$ & $0.15(0.10-0.23)$ & $49(24-101)$ & $0.94(0.01)$ \\
\hline \multicolumn{7}{|l|}{ Ethnicity } \\
\hline Asian & $0.85(0.84-0.87)$ & $0.88(0.87-0.90)$ & $7.61(5.51-10.51)$ & $0.16(0.12-0.21)$ & $61(35-108)$ & $0.94(0.01)$ \\
\hline European & $0.78(0.73-0.83)$ & $0.88(0.80-0.94)$ & $4.83(2.75-8.47)$ & $0.26(0.21-0.33)$ & $21(10-43)$ & $0.85(0.04)$ \\
\hline Overall & $0.84(0.83-0.86)$ & $0.88(0.87-0.90)$ & $7.20(5.40-9.60)$ & $0.18(0.14-0.22)$ & $52(32-85)$ & $0.94(0.01)$ \\
\hline Outliers excluded & $0.71(0.67-0.75)$ & $0.83(0.80-0.87)$ & $4.63(2.85-7.54)$ & $0.35(0.30-0.40)$ & $14(8-26)$ & $0.78(0.03)$ \\
\hline
\end{tabular}

Fig. 3. Summary ROC curves for the dominant subgroups in overall 32 studies. (A) SROC for combined miRNAs (i.e. miRNA panels). (B) SROC for AML patients. (C) SROC for serum-based miRNAs. (D) SROC for Asian population.

\section{A miRnA panels}

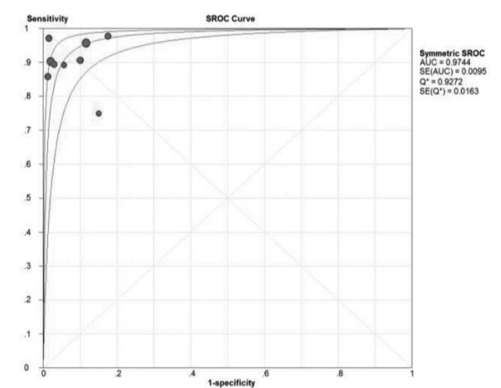

\section{Asian}

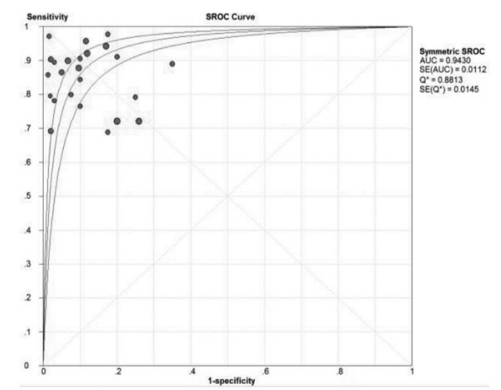

B AML

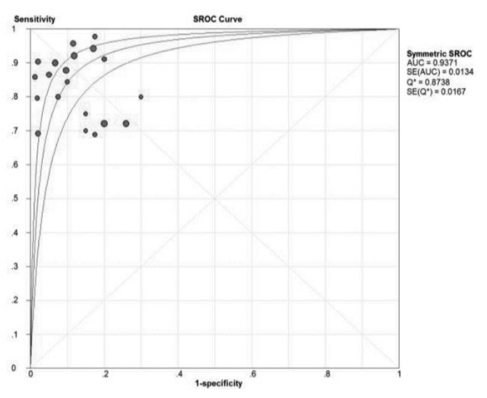

D Serum

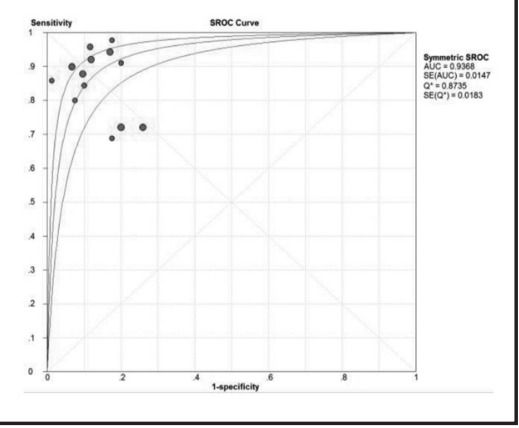

suggested a 4.94 fold higher diagnostic accuracy for combined miRNAs (i.e. miRNA panels) than single ones. After excluding seven studies on single miRNA that only appears once in included studies with lower sensitivity, the overall heterogeneity ( $\mathrm{I}^{2}$ value) decreased from $75.7 \%$ to $45.4 \%$ for DOR. 


\section{A miRNA panels}

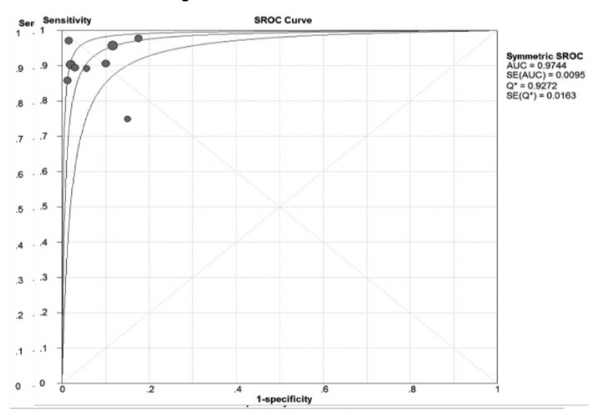

\section{Asian}

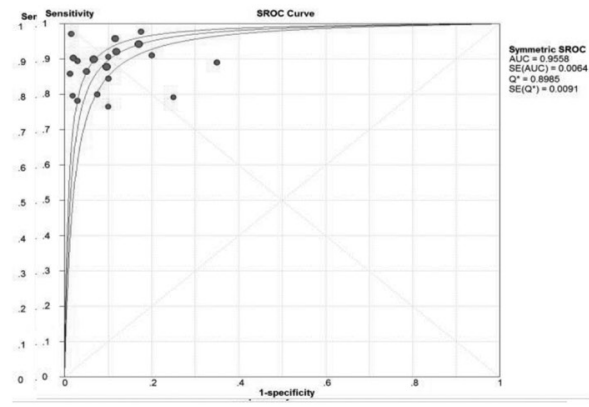

\section{B AML}

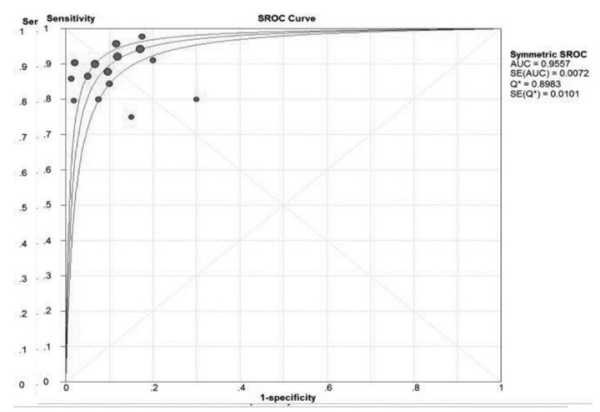

\section{Serum}

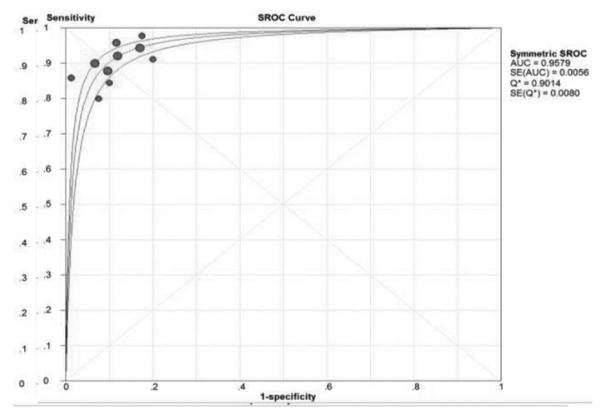

Fig. 4. Summary curves for the dominant subgroups in 25 remaining studies after rejecting 7 outlier studies. (A) SROC for combined miRNAs (i.e. miRNA panels). (B) SROC for AML patients. (C) SROC for serum-based miRNAs. (D) SROC for Asian population.

\section{Subgroup analyses}

Further subgroup analyses also indicated that the diagnostic accuracy was better for combined miRNAs than single ones, with a sensitivity of 0.92 , a specificity of 0.93 , a PLR of 11.53, a NLR of 0.10 , a DOR of 165, and an AUC of 0.97 (Table 2, Fig. 3a). Moreover, acute myeloid leukemia (AML) patients displayed a better performance than other subgroups, with DOR of 51 and an AUC of 0.94 (Fig. 3b). A higher diagnostic value was also found in Asian population compared to European population, with a DOR of 61 and an AUC of 0.94 (Table 2, Fig. 3c). In addition, serum miRNAs have moderately better diagnostic accuracy

than plasma miRNAs for leukemia, with a pooled sensitivity of 0.86 and an AUC of 0.94 (Fig. 3d). Even on the premise of keeping homogeneity, the diagnostic accuracy for remaining 25 studies is still superb (Fig. 4ad).

\section{Publication Bias}

The publication bias of included studies was checked by Deeks' funnel plot asymmetry test, and the result is shown as Fig. 5. A statistically non-significant value $(p=0.75)$ in the funnel plot indicated no potential publication bias, regardless of slight asymmetry due to the small number of publications.

\section{KARGER}

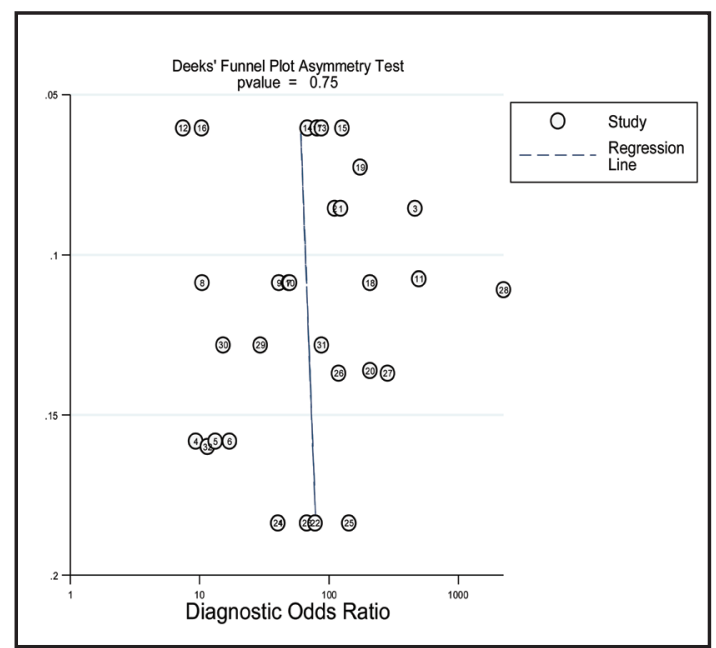

Fig. 5. Funnel plot for the evaluation of potential publication bias of the included studies. 


\section{Cellular Physiology Cell Physiol Biochem 2016;38:939-949 \\ and Biochemistry Published online: March 04, $2016 \quad$\begin{tabular}{l|l} 
DOI: 10.1159/000443046 & $\begin{array}{l}\text { (c) } 2016 \text { The Author(s). Published by S. Karger AG, Basel } \\
\text { www.karger.com/cpb }\end{array}$
\end{tabular} \\ Xu et al.: Circulating MiRNAs in Leukemia Diagnosis}

\section{Discussion}

Leukemia contributed $2.5 \%$ to the overall cancers incidence [1]. Non-invasive and convenient methods for the early detection of leukemia could greatly decrease the worldwide health burden [28], and will be of very useful clinical significance. In the present study, we performed a systematic review pointed to studies related to blood-based circulating miRNAs expression in leukemia. We displayed the most potentially effective candidate circulating miRNAs in diagnosing leukemia, with 14 up-regulated and 6 down-regulated miRNAs, as well as 9 miRNAs combinations. However, it must be noted that significant heterogeneity existed in the data of included studies, which was most likely attributable to the miRNA profile. Subgroup analyses demonstrated that combined miRNA panels were promising biomarkers for leukemia with superb diagnostic accuracy. In addition, AML patients and Asian population presented moderately better performance than other subgroups, respectively. As far as we know, this is the first meta-analysis specially focusing on circulating miRNAs in leukemia, with comprehensively and systemically quantitative evaluation on the diagnostic value.

Circulating miRNAs are packaged inside microvesicles (e.g. exosomes) secreted from cancer cells, and function in new location by being delivered from one cell to another [29, 30]. These might be a class of effective and stable biomarkers, and can be quantitatively measured by qRT-PCR. Kroh et al. [31] have summarized the procedure for qRT-PCR analysis of blood-based circulating miRNAs, which produce increasingly standardized investigation on circulating miRNAs. Since then, circulating miRNAs have attracted a great deal of attention as novel, minimally invasive biomarkers for various diseases. Recently, Li et al. [32] reviewed the publications on circulating miRNAs as diagnostic biomarkers in overall hematologic cancers, and found a better diagnostic accuracy for AML than other cancers in hematopoietic system.

In current meta-analysis, we thoroughly searched multiple databases and retrieved 32 studies aimed at circulating miRNAs for whole leukemia. Analysis showed that blood-based circulating miRNAs has a relatively high diagnostic accuracy in leukemia, with a pooled sensitivity of 0.84 , specificity of 0.88 . Moreover, a PLR value of 7.2 indicated a moderate ability of circulating miRNAs assays in discriminating leukemia patients from normal individuals, and a NLR value of 0.18 also shows a moderate ability to exclude patients without leukemia. Furthermore, DOR value and SROC curve are indexes for overall accuracy. An effective DOR value should be greater than 1 , and higher values suggest better test discrimination [33]. The position of ideal SROC curve is near the upper-left corner, indicating a perfect test [34]. In this meta-analysis, the overall pooled DOR was 52, and AUC of the SROC curve was 0.94, which suggested an excellent diagnostic accuracy of circulating miRNA in leukemia. However, the $\mathrm{I}^{2}$ value of global DOR $>50 \%$, revealing a significant heterogeneity. It is an inevitable issue when interpreting the results for meta-analysis. To explore the potential sources of heterogeneity, we carried out sensitivity analyses. The results of threshold effect and Meta regression analyses showed obvious heterogeneity not caused by threshold ( $p=0.83)$, but originating from miRNAs profile. Then we further searched all the included studies, and found seven studies with lower sensitivity all focused on single miRNA that only appeared once in the included studies. After excluding these seven studies from five publications [16$19,26]$, the heterogeneity ( $\mathrm{I}^{2}$ value) decreased from $75.7 \%$ to $45.4 \%$ for DOR.

Subgroup analyses were conducted according to the classification of disease, miRNAs profile, specimen and ethnicity. Based on miRNAs profile, we showed that combined miRNAs as diagnostic biomarkers for leukemia are more promising, compared to single miRNA, which is consistent with previous studies. Zhou [20] demonstrated that combining miR-150 and miR-155 could be a more powerful diagnostic biomarker for distinguishing AL patients from normal individuals, with a sensitivity of $91.4 \%$ and specificity of $87.5 \%$ than either a single miR-150 with a sensitivity of $77.1 \%$ and specificity of $87.5 \%$, or a single miR-155 with a sensitivity of $88.6 \%$ and specificity of $62.5 \%$. Besides, the results of Moussay et al. also showed that single miRNAs (miR-195, miR-222, or miR-29a) may help rule out individuals without CLL, with $100 \%$ specificity, but a better diagnostic accuracy was obtained by the 
combination of the three miRNAs with increased sensitivity of $90.2 \%$ in the case of specificity unchanged [16]. Moreover, we showed that AML subgroup had a better diagnostic value than other subgroups, similar to the earlier studies. Huang et al. [25] investigated $26 \mathrm{AML}$ patients and 26 healthy controls, revealing that circulating miR-335 was a useful diagnostic biomarker for AML with $80.8 \%$ sensitivity and $100 \%$ specificity. In the study from Xie et al. [19], a high diagnostic value for discriminating AML was present at either single miRNA (miR-21, miR-210, or miR-221) or their combination. In addition, as for ethnicity, it has been reported there existed race-based differences in the amount of circulating miRNAs [32, 35, 36]. We also found higher DOR and AUC value in Asian population compared to European populations (61 versus 21 and 0.94 versus 0.85 , respectively), indicating better accuracy of blood-based circulating miRNAs assays in Asian populations. Taken together, our subgroup analyses showed excellent overall accuracy in miRNAs combination, AML, and Asian subgroup compared to others, which is consistent with the meta-analysis on entire hematological cancers by Li and his colleagues [32]. However, as to the specimen, although both plasma and serum had high diagnostic accuracy in our results, we failed to find an obvious difference between plasma and serum, which is different from the previous studies. McDonald et al. found higher miRNAs concentrations in plasma compared to serum [37], while Li et al. showed a better performance of plasma miRNA assay in diagnosing hematopoietic cancers [32]. In our results, the AUC of serum-based miRNA assays are greater than that of plasmabased miRNA assays (0.94 versus 0.91$)$. In this respect, the overall diagnostic accuracy of serum should be better than plasma, if regardless of the slightly higher DOR in plasma compared to serum (54 versus 49). The two studies on Asian may be able to explain some problems. Chen et al. [13] found that the serum-based miRNAs can keep good concordance with blood cell miRNAs, and Zhang et al. [38] showed no significant difference between plasma miRNAs and serum miRNAs. Thus, to reach at a trade-off, future study should give more attention to the sample source of circulating miRNAs.

Compared to the previous studies, the present meta-analysis has several advantages. Firstly, the objective of this analysis is leukemia's circulating miRNAs. As the first metaanalysis specifically directed at the diagnostic value of circulating miRNAs in leukemia, its results are very encouraging and promising. Secondly, we included more studies on circulating miRNAs by conducting thoughtful search terms to find emerging literatures in recent years. It enhances the reliability of our evaluation on overall diagnostic value of miRNA assay. In addition, we employed Meta-Disc software to quantitatively analyze the previous individual studies. Meta-Disc is a comprehensive and special software for diagnostic metaanalysis and has already been cited in many high-quality meta-analyses, demonstrating the excellent performance of this software [24]. Nevertheless, our meta-analysis still has certain limitations. First, as the value of circulating miRNAs as diagnostic biomarkers has been explored only in recent years, sample sizes have been small, e.g. the study of FayyadKazan et al. [17] included only 20 AML patients. As a result, a small-study effect might be presented. Second, because of relatively fewer studies and smaller sample sizes, subgroup classification on sex, age, and subtype of leukemia, has not been considered in this analysis. Yet these factors are closely associated with the development, progression, and prognosis of certain leukemias [3]. The third, there is no consensus on the endogenous reference for circulating miRNA quantification. Although the detection for circulating miRNAs is all based on qRT-PCR in our studies, different laboratories might take different endogenous controls to quantify circulating miRNAs, which can also contribute to sources of heterogeneity. For example, Fayyad-Kazan et al. selected miR-16, while Tanaka et al. used miR-683 as internal control, since circulating miR-16 and miR-638 have been identified stably and consistently either in patients or in normal individuals $[15,17]$. Unfortunately, most of included studies did not mention the details about the internal control. Finally, our analyses have not covered the functions of circulating miRNAs in ALL and CML, due to absence of eligible studies on these two subtypes.

The current meta-analysis suggested a potential value of blood-based circulating miRNA for diagnosis of leukemia. Through subgroup analysis, we showed better performance in

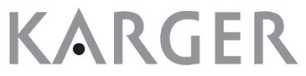




\section{Cellular Physiology Cell Physiol Biochem 2016;38:939-949

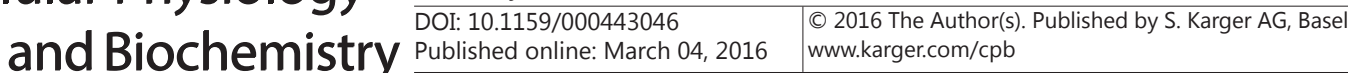 \\ Xu et al.: Circulating MiRNAs in Leukemia Diagnosis}

miRNA panels, AML patients, and Asian population compared to other subgroups. Therefore, miRNA panels can serve as the most potential diagnostic biomarkers in blood-based circulating miRNAs for diagnosis of AML in Asian population. Future studies with a larger sample size are needed to validate these data to promote clinical application.

\section{Acknowledgements}

Lihua Xu, Yang Guo and Xueli Zhang contributed equally to this work. The work was supported by the Natural Science Foundation of China (NO. 81370627 and NO. 81170513), Jiangsu Province key point project (NO. BL2013014), and a Project Funded by the Priority Academic Program Development of Jiangsu Higher Education Institutions.

\section{Disclosure Statement}

The authors declare that they have no conflict of interest.

\section{References}

1 Rodriguez-Abreu D, Bordoni A, Zucca E: Epidemiology of hematological malignancies. Ann Oncol 2007;18:i3-i8.

2 Slater DN: The new World Health Organization classification of haematopoietic and lymphoid tumours: A dermatopathological perspective. Br J Dermatol 2002;147:633-639.

3 Jaffe ES, Harris NL, Stein, HJ, Vardiman, JW: Pathology and Genetics: Tumours of Haematopoietic and Lymphoid Tissues (WHO Classification of Tumours). International Agency for Research on Cancer (IARC) 2001;3:1-351.

4 Kalia M: Biomarkers for personalized oncology: Recent advances and future challenges. Metabolism 2015;64:S16-S21.

5 Lagos-Quintana M, Rauhut R, Lendeckel W, Tuschl T: Identification of novel genes coding for small expressed RNAs. Science 2001;294:853-858.

6 Osada H, Takahashi T: MicroRNAs in biological processes and carcinogenesis. Carcinogenesis 2007;28:212.

7 Cho WC: OncomiRs: The discovery and progress of microRNAs in cancers. Mol Cancer 2007;6:60.

8 Mitchell PS, Parkin RK, Kroh EM, Fritz BR, Wyman SK, Pogosova-Agadjanyan EL, Peterson A, Noteboom J, O‘Briant KC, Allen A, Lin DW, Urban N, Drescher CW, Knudsen BS, Stirewalt DL, Gentleman R, Vessella RL, Nelson PS, Martin DB, Tewari M: Circulating microRNAs as stable blood-based markers for cancer detection. Proc Natl Acad Sci USA 2008;105:10513-10518.

9 Turchinovich A, Weiz L, Langheinz A, Burwinkel B: Characterization of extracellular circulating microRNA. Nucleic Acids Res 2011;39:7223-7233.

10 Madhavan D, Cuk K, Burwinkel B, Yang R: Cancer diagnosis and prognosis decoded by blood-based circulating microRNA signatures. Front Genet 2013;4:116.

11 Shao Y, Geng Y, Gu W, Huang J, Pei H, Jiang J: Prognostic role of tissue and circulating microrna-200c in malignant tumors: A systematic review and meta-analysis. Cell Physiol Biochem 2015;35:1188-1200.

12 Hunter MP, Ismail N, Zhang X, Aguda BD, Lee EJ, Yu L, Xiao T, Schafer J, Lee ML, Schmittgen TD, NanaSinkam SP, Jarjoura D, Marsh CB: Detection of microRNA expression in human peripheral blood microvesicles. PLoS One 2008;3:e3694.

13 Chen X, Ba Y, Ma L, Cai X, Yin Y, Wang K, Guo J, Zhang Y, Chen J, Guo X, Li Q Li X, Wang W, Zhang Y, Wang J, Jiang X, Xiang Y, Xu C, Zheng P, Zhang J, Li R, Zhang H, Shang X, Gong T, Ning G, Wang J, Zen K, Zhang J, Zhang CY: Characterization of microRNAs in serum: A novel class of biomarkers for diagnosis of cancer and other diseases. Cell Res 2008;18:997-1006.

14 Lawrie CH, Gal S, Dunlop HM, Pushkaran B, Liggins AP, Pulford K, Banham AH, Pezzella F, Boultwood J, Wainscoat JS, Hatton CS, Harris AL: Detection of elevated levels of tumour-associated microRNAs in serum of patients with diffuse large B-cell lymphoma. Br J Haematol 2008;141:672-675.

15 Tanaka M, Oikawa K, Takanashi M, Kudo M, Ohyashiki J, Ohyashiki K, Kuroda M: Down-regulation of miR-92 in human plasma is a novel marker for acute leukemia patients. PLoS One 2009;4:e5532.

16 Moussay E, Wang K, Cho JH, van Moer K, Pierson S, Paggetti J, Nazarov PV, Palissot V, Hood LE, Berchem G, 


\section{Cellular Physiology Cell Physiol Biochem 2016;38:939-949

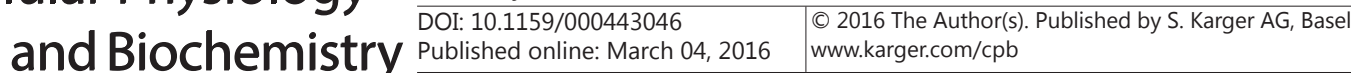 \\ Xu et al.: Circulating MiRNAs in Leukemia Diagnosis}

Galas DJ: MicroRNA as biomarkers and regulators in B-cell chronic lymphocytic leukemia. Proc Natl Acad Sci USA 2011;108:6573-6578.

17 Fayyad-Kazan H, Bitar N, Najar M, Lewalle P, Fayyad-Kazan M, Badran R, Hamade E, Daher A, Hussein N, ElDirani R, Berri F, Vanhamme L, Burny A, Martiat P, Rouas R, Badran B: Circulating miR-150 and miR-342 in plasma are novel potential biomarkers for acute myeloid leukemia. J Transl Med 2013;11:31.

18 Zhi F, Cao X, Xie X, Wang B, Dong W, Gu W, Ling Y, Wang R, Yang Y, Liu Y: Identification of circulating microRNAs as potential biomarkers for detecting acute myeloid leukemia. PLoS One 2013;8:e56718.

19 Xie H, Chu Z, Wang H: Serum microRNA expression profile as a biomarker in diagnosis and prognosis of acute myeloid leukemia. J Clin Pediatr 2012;30:421-424.

20 Zhou Q: The expression and clinical significance of circulating miRNA-150 and miRNA-155 in acute leukemia. Central South University, China. Wanfangdata 2014.

21 Ohyashiki JH, Umezu T, Kobayashi C, Hamamura RS, Tanaka M, Kuroda M, Ohyashiki K: Impact on cell to plasma ratio of miR-92a in patients with acute leukemia: In vivo assessment of cell to plasma ratio of miR92a. BMC Res Notes 2010;3:347.

22 Whiting P, Rutjes AW, Reitsma JB, Bossuyt PM, Kleijnen J: The development of QUADAS: A tool for the quality assessment of studies of diagnostic accuracy included in systematic reviews. BMC Med Res Methodol 2003;3:25.

23 Deville WL, Buntinx F, Bouter LM, Montori VM, de Vet HC, van der Windt DA, Bezemer PD: Conducting systematic reviews of diagnostic studies: Didactic guidelines. BMC Med Res Methodol 2002;2:9.

24 Zamora J, Abraira V, Muriel A, Khan K, Coomarasamy A: Meta-DiSc: A software for meta-analysis of test accuracy data. BMC Med Res Methodol 2006;6:31.

25 Huang S, Lu Y, Liu J: Expression and clinical significance of circulating miR-335 in patients with acute myeloid leukemia. Exp Lab Med 2015;33:147-149.

26 Wang F, Wang XS, Yang GH, Zhai PF, Xiao Z, Xia LY, Chen LR, Wang Y, Wang XZ, Bi LX, Liu N, Yu Y, Gao D, Huang BT, Wang J, Zhou DB, Gong JN, Zhao HL, Bi XH, Yu J, Zhang JW: MiR-29a and miR-142-3p downregulation and diagnostic implication in human acute myeloid leukemia. Mol Biol Rep 2012;39:27132722.

27 Zhou K, Yu Z, Yi S, Li Z, An G, Wang Y, Zou D, Qi J, Zhao Y, Song Y, Qiu L: Expression of microRNA-223 and its clinical value in B lymphoproliferafive disorders. Chinese Medical Journal (Natl Med J China) 2011; 91:2384-2387.

28 Etzioni R, Urban N, Ramsey S, McIntosh M, Schwartz S, Reid B, Radich J, Anderson G, Hartwell L: The case for early detection. Nat Rev Cancer 2003;3:243-252.

29 Valadi H, Ekstrom K, Bossios A, Sjostrand M, Lee JJ, Lotvall JO: Exosome-mediated transfer of mRNAs and microRNAs is a novel mechanism of genetic exchange between cells. Nat Cell Biol 2007;9:654-659.

30 Mears R, Craven RA, Hanrahan S, Totty N, Upton C, Young SL, Patel P, Selby PJ, Banks RE: Proteomic analysis of melanoma-derived exosomes by two-dimensional polyacrylamide gel electrophoresis and mass spectrometry. Proteomics 2004;4:4019-4031.

31 Kroh EM, Parkin RK, Mitchell PS, Tewari M: Analysis of circulating microRNA biomarkers in plasma and serum using quantitative reverse transcription-PCR (qRT-PCR). Methods 2010;50:298-301.

32 Li Q Liu L, Li W: Identification of circulating microRNAs as biomarkers in diagnosis of hematologic cancers: A meta-analysis. Tumour Biol 2014;35:10467-10478.

33 Glas AS, Lijmer JG, Prins MH, Bonsel GJ, Bossuyt PM: The diagnostic odds ratio: A single indicator of test performance. J Clin Epidemiol 2003;56:1129-1135.

34 Walter SD: Properties of the summary receiver operating characteristic (SROC) curve for diagnostic test data. Stat Med 2002;21:1237-1256.

35 Hu Z, Chen X, Zhao Y, Tian T, Jin G, Shu Y, Chen Y, Xu L, Zen K, Zhang C, Shen H: Serum microRnA signatures identified in a genome-wide serum microRNA expression profiling predict survival of non-small-cell lung cancer. J Clin Oncol 2010;28:1721-1726.

36 Zhao H, Shen J, Medico L, Wang D, Ambrosone CB, Liu S: A pilot study of circulating miRNAs as potential biomarkers of early stage breast cancer. PLoS One 2010;5:e13735.

37 McDonald JS, Milosevic D, Reddi HV, Grebe SK, Algeciras-Schimnich A: Analysis of circulating microRNA: Preanalytical and analytical challenges. Clin Chem 2011;57:833-840.

38 Zhang X, Dong J, Ma H, Hu Z, Shen H: Investigation and set up of detection method for serum/plasma-based miRNAs. Acta Universitatis Medicinalis Nanjing (Natural Science) 2011;31:529-531. 